\title{
Observation of the Vortex Lattice Melting by NMR Spin-lattice Relaxation in the Mixed State ${ }^{*}$
}

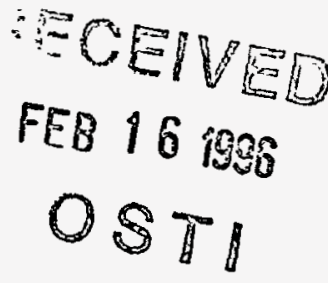

\section{L.N. Bulaevskii, a P.C. Hammel, ${ }^{a}$ and V.M. Vinokur ${ }^{b}$}

aLos Alamos National Laboratory, Los Alamos, NM 87545

bMaterials Science Division, Argonne National Laboratory, Argonne, IL 60439

\section{DISCLAIMER}

jmc

This report was prepared as an account of work sponsored by an agency of the United States Ther employees, makes any warranty, express or implied, or assumes any legal liability or responsibility for the accuracy, completeness, or usefulness of any information, apparatus, product, or bility for the accuracy, compents that its use would not infringe privately owned rights. Referprocess disclosed, or repific commercial product, process, or service by trade name, trademark, ence herein to any specific commercial produst, process, or or imply its endorsement, recommanufacturer, or otherwise dhe views mendation, or favoring by the United States Government or any agencester the of United States Government or any agency thereof.

\section{MASTER}

DISTRIBUTION OF THIS DOCUMENT IS UNLIMITED BS

\footnotetext{
*Work supported by the U.S. Department of Energy, BES-Materials Sciences under contract \#W-31-109-ENG-38.
} 


\title{
Observation of the Vortex Lattice Melting by NMR Spin-lattice Relaxation in the Mixed State
}

December 28, 1993

\author{
L.N. Bulaevskiii ${ }^{a}$, P.C. Hammel ${ }^{a}$, and V.M. Vinokur ${ }^{b}$ \\ "Los Alamos National Laboratory, Los Alamos, NM 87545 \\ b Argonne National Laboratory, Argonne, IL 60499
}

\begin{abstract}
For anisotropic layered superconductors the effect of moving vortices on the nuclear spin magnetization is calculated. Current is supposed to flow along layers, and applied magnetic field is tilted with respect to $c$-axis. In the solid phase the motion of the vortex lattice produces an alternating magnetic field perpendicular to the applied field which causes the decay of the spin-echo amplitude. This decay rate will display an array of peaks as a function of frequency. In the liquid phase this alternating field contribute to the longitudinal relaxation rate $W_{1}$ which has a single peak.
\end{abstract}

The nature of the melting transition in the vortex configuration is now one of the central problems of the physics of the mixed state of high temperature superconductors (HTS). The melting line is commonly related to the sharp drop in resistivity and/or to the peak in ac absorption. It is important to note, however, that these measurements detect actually the abrupt increase in the flux pinning rather than the melting transition itself, and therefore can provide only indirect evidence of the vortex lattice melting. The direct evidence of the vortex lattice melting could be obtained, in principle, from the behavior of the static structure factor which can be provided, for example, by neutron scattering measurements. In this Letter we discuss the transformation of Bragg peaks in the static structure factor into the peaks in the dynamic structure factor of a moving vortex lattice.

The dynamic structure factor can be found much more accurately than the static structure factor by means of the measurements of the NMR spin-lattice relaxation rate. As we will show, NMR techniques have an enhanced sensitivity to the dynamic structure factor relative to the static structure factor and so are well suited to study the moving vortex lattice.

The possibility to probe the motion of the vortex lattice by NMR measurements

was discussed first by Delrieu [1]. He noticed that motion of vortices narrows the inhomogeneous static NMR lineshape. The proposed measurements were successfully realized in CDW systems [2] and with less important results in the case of superconductors. For SDW systems the another method was proposed which is based on the suppression of spin-echo signal due to additional dephasing of nuclei caused by the alternating internal field of moving SDW. This effect can be also characterized as a type of $1 / T_{2}$ measurement [3].

IIere we propose the method to study the structure of moving vortex phase in anisotropic superconductors which can be characterized as measurements of the decay of the spin-echo amplitude and the NMIR relaxation 
rate $W_{1}=1 / T_{1}$. It is based on a transformation of the Bragg peaks of the vortex lattice in the frame moving wilh vortices into the alternating periodic magnetic field in the laboratory frame.

$\Lambda$ key point is that in the anisotropic superconductors, if the applied field is tilted away from the $c$-axis, the alternating field will have a component perpendicular to the applied field. This alternating field causes either precession of the nuclear magnetization or longitudinal relaxation depending on the state of the vortex lattice. The corresponding rate of precession or relaxation as function of NMR frequency and vortex velocity $\mathbf{v}$ has series of peaks in the ordered lattice phase corresponding to the Bragg peaks, and only one peak in the disordered (melted) vortex phase. Thus the structure and melting of moving vortex lattice can be studied by the standard NMR spin-echo technique.

We notice first that magnetic field inside superconductor in the vortex phase at rest has an inhomogeneous spatially periodic component: it is higher near vortex cores and weaker in the space between vortices. In the isotropic superconductors this field is aligned with the applied field $\mathrm{H}_{0}$ and is given as

$\mathbf{h}(\mathbf{r})=\sum_{\mathbf{G}} \mathbf{h}(\mathbf{G}) \exp (i \mathrm{Gr}), \quad h(\mathrm{G})=\frac{B}{2 \pi^{2}\left(1+\lambda_{a b}^{2} G^{2}\right)}$,

where $\mathbf{G}$ are reciprocal vectors of vortex lattice and $\lambda_{a b}$. is the London penetration length for currents along $a b$ plane [4]. At large applied fields, $H_{0} \gg H_{c 1}$, the spatial modulation of the field

inside a superconductor is of the order of lower critical field, $H_{c 1}$. The key point is that in highly anisotropic layered superconductors like all HTS materials $h(\mathbf{r})$ is oriented practically perpendicular to the layers (along caxis) at any orientation of the applied field $\mathrm{H}_{0}$. Thus in the layered superconductor if the applied field $\mathrm{H}_{0}$ is tilted with respect to $c$-axis, the inhomogeneous component of the field is tilted in the same way with respect to the homogeneous one.

Now, if the lattice is moving, the inhomogeneous component of the field gives rise to a periodic alternating field

$$
\mathrm{h}(\mathrm{r}, t)=\sum_{\mathrm{G} \neq 0} \mathrm{~h}(\mathrm{G}) \exp [i \mathrm{G}(\mathrm{r}-\mathrm{v} t)],
$$

with frequencies $\omega(G)=G v$. In an anisotropic superconductor this alternating field has a component, perpendicular to $\mathrm{H}_{0}$, which causes nutation of nuclear magnetization in the plane formed by $\mathrm{H}_{0}$ and $\mathrm{h} \times \mathrm{H}_{0}$ in spinecho experiments. Its effect is strong when the NMIR frequency $\omega=\gamma_{n} H_{0}$ is close to $\omega(G)$, here $\gamma_{n}$ is the gyromagnetic ratio. In the following we will discuss the case of very strong anisotropy. Then the alternating field is oriented predominantly along $c$-axis. 
Let as denote by $\alpha$ the angle between $\mathrm{H}_{0}$ and $c$-axis. We choose the coordinate system with $z$ along $c$-axis, $y$ along projection of $\mathrm{H}_{0}$ on $a b$ plain, $z^{\prime}$ along $\mathrm{H}_{0}, y^{\prime}$ lies in the $\mathrm{c}, \mathrm{H}_{0}$ plain perpendicular to $\mathrm{H}_{0}$, and $x=x^{\prime}$ is perpendicular to $y, z$, see Fig. .

We will discuss two regimes. In the case of an ordered vortex lattice, the spatial periodicity of the static lattice will produce an alternating field for the nuclear spins. The component of this which is perpendicular to the applied field, $h_{\perp}=h \sin \alpha$, will cause nutation of the nuclear spins in $x, z^{\prime}$ plane with frequency determined by strength of the varying field, $\Omega(\mathbf{r})=$ $\gamma_{n} h(\mathbf{G}) \sin \alpha \cos (\mathbf{G r})$. Note, that $\Omega$ depends on the coordinates of nuclei inside vortex unit cell.

In a spin-echo experiment, applied rf pulses produce rotations of the nuclear magnetization $M$ about the rf field. An initial rotation of 90 degrees leaves $M$ in the $x-y^{\prime}$ plane where it dephases due to inhomogeneities in the environment of the nuclear spin. A subsequent 180 degree pulse produces a situation in which the coherence of the nuclear spins occurs producing a so-called spinecho. In the present case, the application of a pulse of current will cause the lattice to move and thus produce an alternating field similar to the rf field applied by the NMR coil. If this field were homogeneous throughout the sample and were inserted between the 90 degree and 180 degree pulses it would periodically modulate the amplitude of the resulting spin-echo. This modulation would be periodic in the length of the applied current pulse with a period determined by the strength of the variation in the field from the vortex lattice. Note however that the nutation frequency $\Omega$ due to the current pulse depends on the nuclear position in the unit cell of the vortex lattice producing a different frequency for different nuclear spins. This inhomogeneity will cause a rapid decay of this otherwise periodic modulation of the echo intensity with current pulse length. We will discuss only the initial, quadratic time dependence, proportional to $\left(W t_{p}\right)^{2}$, of the echo amplitude on current pulses length $t_{p}$. In the case of a disordered (melted) vortex lattice the alternating fields generated by the moving lattice will produce a randomly fluctuating field and thus will contribute to the nuclear spin lattice relaxation rate, $W_{1}$. This situation will be discussed below.

The suppression of the echo will be peaked at NMR frequencies $\omega=\mathrm{Gv}$. For a perfectly ordered lattice the widths of the peaks will be given the NMR lineshape $f(\omega)$. Ilowever, as in the case of usual crystal lattices, disorder and thermal fluctuations suppress the intensity of the Bragg peaks. For our system, the same effects suppress $h(G)$ and the peaks in $W(\omega)$. Thus, we introduce the corresponding Debye-Waller factor $g(\omega)$ which will be estimated later. Finally the frequency of precession of the magnetization vector in the ordered lattice is

$$
W(\omega) \approx \gamma_{n} h(G) f(\omega) g(\omega) \sin \alpha .
$$


For minimum reciprocal vector, $G_{0} \approx 2 \pi\left(H_{0} / \Phi_{0}\right)^{1 / 2}$, resontance occurs at velocity $v \approx \gamma_{n}\left(H_{0} \Phi_{0}\right)^{1 / 2}$. For ${ }^{205} \mathrm{Tl}$ unclei with $\gamma_{n}=2.45 \cdot 10^{3} \mathrm{~Hz} / \mathrm{G}$ this resonance velocity is $\approx 100 \mathrm{~cm} / \mathrm{sec}$ in a $1 \mathrm{~T}$ field. We can estimate $h(\mathrm{G}) \sin \alpha$, with help of Eq. (1), for fields well above $H_{c 1}$ to be $\approx \Phi_{0} / 8 \pi^{2} \lambda_{a b}^{2} \approx 10 \mathrm{G}$. This value provides very high nutation rate $W \approx 2.5 \times 10^{4} \mathrm{sec}^{-1}$ at $g(\omega) \approx 1$.

To express $v$ via the applied current, we suppose that when current with density $j$ flows in $a b$ plane along $y$ axis, it results in motion of vortices along $x$-axis in $a b$ plane. At high currents, in the flux-flow regime, their velocity $v_{x}$ is determined by equation of viscous motion, $\eta v_{x}=\Phi_{0} j / c$, where $\eta$ is viscosity coefficient per unit length of vortex. In $\mathrm{Bi}(2: 2: 1: 2)$ at $T<70 \mathrm{~K}$ the viscosity coefficient is $\eta \leq 10^{-7} \mathrm{~g} / \mathrm{cm} \cdot \mathrm{s}$ [5], $j$ will be then in the interval $\left(10-10^{6}\right) \mathrm{A} / \mathrm{cm}^{2}$. This gives $v$ in the interval $(10$ $10^{6}$ ) $\mathrm{cm} / \mathrm{s}$. At $B=1 \mathrm{~T}, j=10 \mathrm{~A} / \mathrm{cm}^{2}$ we get $\omega \approx 3 \cdot 10^{6}$ $\mathrm{Hz}$. The frequency $\omega$ varies as $\sqrt{B}$, and increases linearly with $j$. The distance between peaks is approximately $\omega$. Thus, for perfect moving lattice, Bragg peaks are convert into peaks in the nutation rate $W$.

Let us consider now the relaxation rate $W_{1}$ in the presence of disordered moving lattice. Above melting temperature $T_{m}$ the vortex lattice at rest or in motion is in a liquid state. In the moving liquid vortex phase the time dependent component of the magnetic field, $h_{z}(t)$ is a random function. Then we obtain the relaxation rate $W_{1}$ for nuclei at position $r$ :

$$
W_{1}(\omega)=\frac{1}{2} \gamma_{n}^{2} \int d t\left\langle h_{z}(\mathbf{r}, t) h_{z}(\mathbf{r}, 0)\right\rangle \exp (i \omega t),
$$

where $\langle\ldots\rangle$ means thermodynamic and disorder averaging. The field $h_{z}(t)$ may be expressed via the structure factor of the vortices. For highly anisotropic superconductors with Josephson-coupled layers (like Bi- and Tlcompounds) we use the pancake model for vortices [6]. We denote by $\mathbf{r}_{n v}$ coordinates of two-dimensional pancake vortices in the layer $n, \nu$ is a set of two integer numbers which label the vortices. Moving vortices induce a time varieng magnetic field [7]:

$$
\begin{gathered}
h_{z}(\mathrm{r}, z, t)=\frac{\Phi_{0} s}{4 \pi^{3}} \sum_{n, \nu} \int_{-\infty}^{+\infty} d q \int_{-\infty}^{+\infty} \frac{d \mathbf{k}}{1+\lambda_{a b}^{2}\left(k^{2}+Q^{2}\right)} \times \\
\frac{k^{2}+Q^{2}}{k^{2}+q^{2}} \exp \left\{i \mathrm{k}\left[\mathbf{r}-\mathbf{r}_{n \nu}(t)\right]+i q(z-n s)\right\}
\end{gathered}
$$

where $Q^{2}=2(1-\cos q s) / s^{2}$ and $s$ is the interlayer spacing. With help of Eqs. (4), (5) we get the relaxation rate averaged over nuclear positions in the vortex unit cell:

$$
\begin{aligned}
\widetilde{W}_{1}(\omega)= & \frac{s \Phi_{0} \gamma_{n}^{2} B}{4 \pi^{3}} \int_{0}^{2 \pi / s} d q \int d \mathrm{k} \frac{S(\mathrm{k}, q, \omega)}{\left[1+\lambda_{a b}^{2}\left(k^{2}+Q^{2}\right)\right]^{2}} \times \\
& \left(\frac{k^{2}+Q^{2}}{k^{2}+q^{2}}\right)^{2}
\end{aligned}
$$

where 


$$
\begin{aligned}
& S(\mathbf{k}, q, \omega)=\frac{1}{N} \sum_{n, \nu, n^{\prime}, \nu^{\prime}} \int d t \exp (i \omega t) \times \\
& \left\langle\exp \left\{-i \mathbf{k}\left[\mathbf{r}_{n \nu}(t)-\mathbf{r}_{n^{\prime} \nu^{\prime}}(0)\right]-i q\left(n-n^{\prime}\right) s\right\}\right\rangle
\end{aligned}
$$

is the structure factor and $N$ is the number of pancakes. We assume that pancakes in different layers are uncorrelated and the correlation function inside a layer in the moving frame is $S(k, t)=S(k, 0) \exp (-\delta t)$. For small $\delta$ we get:

$\bar{W}_{1}(\omega)=\frac{\gamma_{n}^{2} B \Phi_{0} s \omega}{16 \pi^{2} \lambda_{a b} v^{2}} \times$

$$
\int_{1}^{\infty} d x \frac{x S(\omega x / v)}{\left(1+\lambda_{a b}^{2} \omega^{2} x^{2} / v^{2}\right)^{3 / 2}\left(x^{2}-1\right)^{1 / 2}} .
$$

Assuming that the function $S(k, 0)$ has broad peak near $k \approx G_{0}$ and smooth behavior at $k \rightarrow 0$, we obtain the maximum $\bar{W}_{1}$ at very low frequency, $\omega \approx \omega_{p}=$ $v / \lambda_{a b}$, due to superconducting screening. This screening strongly suppresses the ac magnetic field at frequencies higher than $v / \lambda_{a b}$, and the broad structural peak in $S(k, 0)$ becomes invisible. We get

$$
\bar{W}_{1}\left(\omega_{p}\right) \approx \frac{\gamma_{n}^{2} B \Phi_{0} s}{16 \pi^{2} \lambda_{a b}^{2} v} .
$$

It is smaller than the peak at $\omega=G_{0} v$ in the solid phase by factor $\gamma_{n} H_{0} s / v$, which is of the order $0.01-0.1$ for fields $0.1-1 \mathrm{~T}$. Thus in the liquid phase one weak peak should exist at very low frequency in contrast to the solid phase where series of peaks can be observed at much higher frequencies, $\omega_{n} \approx G_{0} v n$.

The state of the moving vortex lattice depends on temperature, pinning and current density. Below the irreversibility line and at low currents the lattice is solid and pinned, this implies that pinning destroys the long range order of the static vortex lattice [10] and that the vortex motion occurs via the thermal activation of the vortex bundles over pinning barriers. Above a certain critical value of the current density, $j_{c}$, the lattice becomes unpinned and displays viscous motion although it is still affected by pinning. Namely, in the interval $j_{c}<j<j_{a}$ the long-range order in the lattice is still destroyed by pinning, and the vortex lattice motion can reveal features of the plastic flow [13]. At still higher currents, $j>j_{a}$, pinning becomes ineffective $\left(B>1 \mathrm{~T}\right.$ and $j>2000 \mathrm{~A} / \mathrm{cm}^{2}$ in $\mathrm{Bi}$ 2:2:1:2), and lattice recovers its long-range order and moves coherently as a whole. In the frame moving with the vortex lattice, vortices are still affected by an alternating force caused by disorder although the effect of this force drops with velocity of a moving lattice. Locally the collisions of the vortex lines with the pinning centers give rise to the effect analogous to that of the thermal Langevin force. In the lowest order of perturbation theory with respect to disorder the pinning potential causes additional distortions in the moving vortex lattice analogous to those caused by finite temperature. 
On the basis of the Lindemann criterion one can conclude that there exists a certain current, $j_{a}>j_{c}$, and corresponding velocity, $v_{\alpha}>v_{c}$, above which vortex lattice recovers its long-range order [13], and in what follows we will estimate $v_{\alpha}$. In this state the width of the peaks in $W_{1}(\omega)$ are determined by NMR line $f(\omega)$ but the intensity of the peaks is suppressed due to a Debye-Waller factor caused by disorder and thermal fluctuations. At $v<v_{\alpha}$ "Bragg" peaks in $\bar{W}_{1}$ are absent (in strongly disordered liquid phase) or they are broadened by disorder in the phase with large correlation length.

Let us estimate $v_{\alpha}$ and calculate the disorder induced Debye-Waller factor for a moving lattice. We present the coordinates of the $(n, \nu)$-th vortex as $\mathbf{r}_{n \nu}(t)=\mathbf{r}_{n \nu}^{0}+$ $u_{t h, n \nu}(t)+u_{p, n \nu}(t)+v t$, where $u_{t h}$ are the thermal distortions and $u_{p}$ are disorder induced distortions $[8,13]$. We get $u_{t h}^{2} \approx 16 \pi \gamma \lambda_{a b}^{2} / \Phi_{0}^{3 / 2} B^{1 / 2}$, where $\gamma$ is the anisotropy parameter $[8,9]$. The value $u_{p}^{2}$ determines the disorder induced distortions:

$$
u_{p}^{2}=\lim _{t \rightarrow \infty}\left\langle\left[u_{p, n \nu}(t)-u_{p, 00}(0)\right]^{2}\right\rangle_{d},
$$

if this limit exists. $u_{p}(t)$ can be considered as distortions induced by moving disorder in the frame which moves with moving vortex lattice. One gets:

$$
\begin{aligned}
& u_{p, n \nu}^{\alpha}(\omega)=\sum_{n^{\prime} \nu^{\prime}} G^{\alpha \beta}\left(\mathbf{r}_{n \nu}-\mathbf{r}_{n^{\prime} \nu^{\prime}}, \omega\right) F_{p, n^{\prime} \nu^{\prime}}^{\beta}(\omega), \\
& G_{\alpha, \beta}(\mathbf{k}, q, \omega) \approx \frac{\delta_{\alpha, \beta}-k_{\alpha} k_{\beta} / k^{2}}{\epsilon_{t}(\mathbf{k}, q)-i \eta \omega} .
\end{aligned}
$$

Here $G^{\alpha \beta}$ is the response function, $\epsilon_{t}(\mathbf{k}, q)=c_{66} k^{2}+$ $c_{44} Q^{2}$ is elastic energy of transversal distortions, $c_{66}$ and $c_{44}$ are shear and tilt moduli, see [9]. We neglect here the effect of longitudinal distortions because elastic energy of these distortions is larger than of transverse ones. $F_{p}^{\alpha}$ is the pinning force related to the pinning potential $U_{p}(r)$ as:

$$
\mathbf{F}_{p, n \nu}=-\sum_{m, \mu} U_{p}\left(\mathbf{r}_{n \nu}-\mathbf{r}_{m \mu}\right) \frac{\partial p\left(\mathbf{r}-\mathbf{r}_{m \mu}\right)}{\partial \mathbf{r}_{m \mu}},
$$

where $p(\mathbf{r})$ is the form factor of pancake vortex. Averaging over disorder we obtain:

$$
\begin{aligned}
u_{p}^{2} & =\frac{\gamma_{U} B^{2}}{2(2 \pi)^{3} \Phi_{0}^{2}} \sum_{\mathrm{G}} \int d \mathbf{k} d q \frac{G^{2}|p(\mathrm{G})|^{2}}{\eta^{2}(\mathrm{Gv})^{2}+\epsilon_{t}^{2}(k, q)} \\
& \approx \frac{\gamma_{U} G_{0}^{3 / 2}}{c_{6 \sigma}\left(\eta v \tilde{c}_{44}\right)^{1 / 2}},
\end{aligned}
$$

where $p(\mathrm{G})$ is the Fourier component of the form factor, and the parameter $\gamma_{U}$ characterizes disorder: $\left\langle U_{p}(\mathbf{r}) U_{p}\left(\mathbf{r}^{\prime}\right)\right\rangle=\gamma_{U} \delta\left(\mathbf{r}-\mathbf{r}^{\prime}\right)$. Notice that $u_{p}^{2}$ is finite at $v>0$ contrary to the result [10] at $v=0$. As we mentioned above, this means that the effect of disorder on moving lattice is weaker, and there is critical value of the 
velocily $v_{x}$ above which lattice remains ordered. We estimate it using the Lindemann criterion $u_{p}=c_{L} a_{0}$, where $c_{L} \approx 0.15$ and $a_{0}=\left(\Phi_{0} / B\right)^{1 / 2}$ is the spacing between vorlices.

For moderate magnetic fields

$u_{p}^{2} \approx a_{0}^{2} 10^{2} \frac{\lambda_{a b}}{\xi_{a b} \gamma}\left(\frac{j_{c}}{j_{0}}\right)^{3 / 2}\left(\frac{j_{0}}{j}\right)^{1 / 2}\left(\frac{\xi_{a b}}{a_{0}}\right)^{1 / 2}$.

IIere $j_{0}=c H_{c} / 3 \sqrt{6} \lambda_{a b}$ is the pair-breaking current. The current $j_{\alpha}$ at which $v=v_{\alpha}$ usually lies somewhere above the critical current $j_{c}$ and is

$j_{a}=j_{c} \frac{10^{4}}{c_{L}^{4}}\left(\frac{\lambda_{a b}}{\xi_{a b} \gamma}\right)^{2}\left(\frac{j_{c}}{j_{0}}\right)^{2} \frac{\xi_{a b}}{a_{0}}$.

Using the typical values of parameters we find that $j_{a} \approx$ $10 j_{c}$ at fields of about $1 \mathrm{~T}$. At $j>j_{a}$ sharp peaks in NMR relaxation rate corresponding to the Bragg peaks are expected. The disorder induced distortions reduce these peaks by factors $g\left(\omega_{n}\right)=\exp \left[-u_{p}^{2} G_{0}^{2} n^{2}\right)$, where $n$ is the number of peak. Since at moderate field

$$
u_{p}^{2} G_{0}^{2} \simeq 10 \frac{\lambda_{a b}}{\xi_{a b} \gamma}\left(\frac{\xi_{a b}}{a_{0}}\right)^{1 / 2}\left(\frac{j_{a}}{j}\right)^{1 / 2}
$$

we see that at currents near $j_{a}$ disorder can suppress the Bragg-like structure of frequency dependence of NMR relaxation. For $\mathrm{Bi}(2: 2: 1: 2) \lambda_{a b} / \xi_{a b \gamma} \leq 0.5$ and we get $u_{p} G_{0} \approx 1$ in the field $1 \mathrm{~T}$ and $j \geq j_{a}$.

In conclusion, we have shown that the melting of the moving vortex lattice vortex lattice is marked by the disappearance of the Bragg like peaks structure in the frequency dependence of the NMR spin-lattice relaxation rate. In the solid we expect the series of peaks corresponding to the Bragg peaks, whereas in the liquid state only one low frequency weak peak should exist. The disorder gives rise to the broadening of these peaks in the solid state at low currents, $j<j_{a}$, (this reflects the loss of the long range order in the vortex solid), and to the reducing of these peaks by the Debye-Waller factor at $j>j_{a}$.

The authors thanks M.P. Maley for useful discussion. This work was supported by the U.S. Department of Energy. VMV acknowledges support from Argonne National Laboratory through U.S. Department of Energy, BES-MLaterials Sciences, under contract No W-31-109ENG-38.

[1] J-.H Delrieu, J. Phys. F: Metal Phys., 3, 893 (1973).

[2] J.H. Ross, Z. Wang, and C.P. Slichter, Phys. Rev. B, 41, 2722, (1990). 
[3] W.G. Clark, M.E. Hanson, W.H. Wong, and B. Alavi, Europhys. Lett., (1993).

[4] E.H. Brandt, J. Low Temp. Phys. 73, 355 (1988).

[5] L.N. Bulaevskii, J.H. Cho, M.P. Maley, P. Kes, Qiang Li, M. Suenaga, and M. Ledvij, preprint, 1993.

[6] J.R. Clem, Phys. Rev. B 43, 7837 (1991); A. Buzdin and D. Feinberg, J. Phys. (Paris) 51, 1957 (1990).

[7] Y.-Q. Song, W.P. Halperin, L. Tonge, T.J. Marks, M. Ledvij, V.G. Kogan, and L.N. Bulaevskii, Phys. Rev. Lett. 70, 3127 (1993).

[8] M. V. Feigelman and V. M. Vinokur, Phys. Rev. B 41, 8986 (1990).

[9] L.I. Glazman and A.E. Koshelev, Phys. Rev. B 43, 2835 (1991).

[10] A.I. Larkin, Zh. Eksp. Teor. Fiz. 58, 1466 [Sov. Phys. JETP 31, 784 (1970)].

[11] A.I. Larkin and Yu.N. Ovchinnikov, Zh. Eksp. Teor. Fiz. 65, 1704 [Sov. Phys. JETP 38, 854 (1974)].

[12] G. Blatter, M.V. Feigel'man, V.B. Geshkenbein, A.I. Larkin, V.M. Vinokur, preprint, 1993.

[13] A.E. Koshelev and V.M. Vinokur, preprint, 1993.

The coordinate system and the geometry of the proposed spin-echo experiment. 